\title{
ON REDUCIBILITY BY RECURSIVE FUNCTIONS
}

PAUL R. YOUNG ${ }^{1}$

Throughout this paper, the word "set" means set of nonnegative integers.

Definition (Kleene-Post). The join of two sets $A$ and $B$ $(J(A, B))$ is $\{2 x \mid x \in A\} \cup\{2 x+1 \mid x \in B\}$.

1. Introduction. In [5], Kleene and Post show that $J(A, B)$ determines a least upper bound of the degrees of $A$ and $B$ in the partial ordering of Turing degrees of unsolvability. It is easily shown (and well known) that $J(A, B)$ determines a least upper bound of the degrees of $A$ and $B$ in the partial orderings of truth-table degrees, bounded-truth-table degrees, and many-one degrees. Thus the partial orderings of these degrees are all upper semi-lattices. The principal result of this paper (Corollary 2) is that the partial ordering of oneone degrees is not an upper semi-lattice. A second result is that there is a pseudocreative set $P$ and a simple set $S$ such that $P$ is many-one reducible to $S$ but $S$ is not Turing reducible to $P$.

2. Notation. We write $A \leqq_{1} B$ to indicate that there is a 1-1 recursive function $f$ such that $x \in A$ if and only if $f(x) \in B$, and we write $A \$_{1} B$ if there is no such function. If both $A \leqq_{1} B$ and $B \leqq_{1} A$, we write $A \equiv_{1} B$, and if neither $A \leqq_{1} B$ nor $B \leqq_{1} A$ we say that $A$ and $B$ are 1-1 incomparable. We write $A \leqq_{m} B$ if there is some recursive function $g$ such that $x \in A$ if and only if $g(x) \in B$. If $A \leqq_{m} B$ and $B \leqq_{m} A$, we write $A \equiv_{m} B . N$ is the set of all nonnegative integers and if $A$ is a set, $A^{\prime}=N-A$.

\section{The 1-1 reducibility ordering is not an upper semi-lattice.}

Lemma 1. If $A$ and $B$ are simple sets, so is $J(A, B)$.

Proof. Clearly $J(A, B)$ is recursively enumerable (r.e.). Since $A \leqq_{1} J(A, B)$ and $A$ is not recursive, $J(A, B)$ cannot be recursive. Therefore if $J(A, B)$ is not simple, there is some infinite r.e. set $C \subset J(A, B)^{\prime}$. But in this case, either $\{x \mid 2 x \in C\}$ is an infinite r.e. subset of $A^{\prime}$ or $\{x \mid 2 x+1 \in C\}$ is an infinite r.e. subset of $B^{\prime}$. Since both $A$ and $B$ are assumed to be simple, either is a contradiction, and we conclude that $J(A, B)$ must be simple.

Presented to the Society, April 29, 1963; received by the editors June 26, 1963.

${ }^{1}$ Supported by a National Science Foundation Fellowship. The results reported here are a portion of the author's doctoral dissertation written at the Massachusetts Institute of Technology under the direction of Professor Hartley Rogers, Jr. 
Corollary 1. If two simple sets, $A$ and $B$, have a 1-1 least upper bound $L$, then $L$ is simple.

Proof. Clearly $L$ is not recursive. Since $L$ is assumed to be a 1-1 least upper bound for $A$ and $B$ and since $J(A, B)$ is a 1-1 upper bound for $A$ and $B, L \leqq_{1} J(A, B)$. This shows that $L$ must be r.e. Let $f$ be a 1-1 recursive function such that $x \in L$ if and only if $f(x) \in J(A, B)$. Since $L$ is not recursive, if $L$ were not simple there would be an infinite r.e. set $C \subset L^{\prime}$. But then $f(C)$ would be an infinite r.e. subset of $J(A, B)^{\prime}$, contradicting the fact that $J(A, B)$ is simple. Therefore if $L$ is a least upper bound, $L$ is simple.

Lemma 2 (Tennenbaum). If $A$ and $B$ are infinite r.e. sets and if $A \leqq_{m} B$ via the recursive function $g$ and if $g$ is $1-1$ on $A^{\prime}$, then there exists a 1-1 recursive function $f$ such that $f(A)=B$ and $x \in A^{\prime}$ implies that $f(x)=g(x)$.

Proof. See [2, p. 70].

In [2], Dekker and Myhill show that if $A$ is an immune set and $x \in A^{\prime}$, then $A \cup\{x\} \$_{1} A$.

Lemma 3 (DekKer-Myhill). If $S$ is a simple set and $x \in S^{\prime}$, then (i) $S \cup\{x\} \equiv_{m} S$, (ii) $S \cup\{x\} \varliminf_{1} S$, and (iii) $S \varliminf_{1} S \cup\{x\}$.

Proof OF (i) AND (ii). Let $R$ be an infinite recursive subset of $S$ and let $g$ be a 1-1 recursive function whose range is $R$. Define

$$
\begin{aligned}
& f(x)=g(0), \\
& f(y)=g(n+1) \text { if } y=g(n), \\
& f(y)=y \text { if } y \notin R \text { and } y \neq x .
\end{aligned}
$$

Then $S \cup\{x\} \leqq_{1} S$ via $f$.

Let $z$ be any member of $S^{\prime}$ other than $x$. Define $h(x)=z$ and $h(y)=y$ unless $y=x$. Then $S \leqq_{m} S \cup\{x\}$ via $h$.

Proof of (iii). Suppose $S \leqq_{1} S \cup\{x\}$ via some 1-1 recursive function $f$. Then $\left\{x, f(x), f^{2}(x), f^{3}(x), \cdots\right\}$ is an infinite r.e. subset of $S^{\prime}$, contradicting the assumption that $S$ is simple.

Theorem 1. Suppose $S_{0}$ and $S_{1}$ are 1-1 incomparable simple sets. If $L^{*}$ is a simple 1-1 upper bound for $S_{0}$ and $S_{1}$, then there is an infinite collection of simple sets, $\left\{\cdots, L_{-2}, L_{-1}, L_{0}, L_{1}, \cdots\right\}$, such that

(i) $L^{*}=L_{0}$,

(ii) $L_{i} \leqq{ }_{1} L_{j}$ for all $i \leqq j$,

(iii) $L_{j} \$_{1} L_{i}$ for any $i<j$,

(iv) $L_{i} \equiv_{m} L_{j}$ for all $i$ and $j$, and

(v) $S_{0} \leqq_{1} L_{i}$ and $S_{1} \leqq_{1} L_{i}$ for all $i$. 
Proof. Note that if $S$ is a simple set and $y \in S$, then we may employ Lemma 3 to obtain $S \leqq_{1} S-\{y\}$. Thus from the transitivity of 1-1 reducibility and from Lemma 3 , we see that it suffices to show that if $L$ is any simple 1-1 upper bound for $S_{0}$ and $S_{1}$ then there is an integer $x_{0} \in L^{\prime}$ such that both $S_{0}$ and $S_{1}$ are 1-1 reducible to $L \cup\left\{x_{0}\right\}$. Therefore assume that $L$ is a 1-1 upper bound for $S_{0}$ and $S_{1}$ and that $S_{0} \leqq_{1} L$ via $f_{0}$ and $S_{1} \leqq_{1} L$ via $f_{1}$. Applying Lemma 2 , we may assume that $f_{0}\left(S_{0}\right)=L=f_{1}\left(S_{1}\right)$.

There exists $x_{0} \in L^{\prime}-$ range $f_{0}$ and there exists $x_{1} \in L^{\prime}-$ range $f_{1}$, because, for example, if no such $x_{0}$ exists, $f_{0}$ is a recursive permutation and hence $S_{0} \equiv_{1} L$ and $S_{1} \leqq_{1} S_{0}$, a contradiction.

Since $x_{0} \notin$ range $f_{0}, S_{0} \leqq_{1} L \cup\left\{x_{0}\right\}$ via $f_{0}$. Define

$$
h(x)=f_{1}(x) \text { if } f_{1}(x) \neq x_{0}, \quad h(x)=x_{1} \text { if } f_{1}(x)=x_{0} .
$$

Then $x_{0} \notin$ range $h$ and $S_{1} \leqq_{1} L \cup\left\{x_{0}\right\}$ via $h$.

Corollary 2. No two 1-1 incomparable simple sets have a common 1-1 least upper bound. The partial ordering of 1-1 degrees is not an upper semi-lattice.

Proof. The first assertion follows from Corollary 1, which asserts that any such least upper bound would have to be simple, and from the preceding theorem, which shows that no simple set can be such a least upper bound.

To prove the second assertion it suffices to show that 1-1 incomparable simple sets exist. But by Friedberg's solution to Post's Problem [3], there exist Turing incomparable r.e. sets, and by a result of Dekker [1], every nonrecursive r.e. set is Turing equivalent to some hypersimple set. Thus Turing incomparable hypersimple sets exist, and hence 1-1 incomparable simple sets exist.

4. Structure of r.e. sets. R.e. sets have been classified by the richness of their complements in the possession of r.e. subsets. (See [7] and [8].) For example, a noncreative r.e. set $A$ has been called pseudocreative if for every r.e. set $B \subset A^{\prime}$ there exists an infinite r.e. set $C \subset A^{\prime}$ such that $B \cap C=\varnothing$. In [6], Myhill points out that if $A$ is any nonrecursive noncreative r.e. set and if $B$ is recursively isomorphic to $\{(a, n) \mid a \in A$ and $n \in N\}$, then $B$ is pseudocreative. Such a set, $B$, is called a cylinder of $A$, and it is readily verified that if $B$ is a cylinder of $A$ then $A \leqq_{1} B$ and $A \equiv_{m} B$.

In [9], the author has studied relations between one such classification of the r.e. sets and $1-1$ and $m-1$ reducibility. For those reducibilities at least as strong as $m-1$ reducibility and no stronger than Turing reducibility, the following theorem shows one difficulty in relating the richness of the complements of two r.e. sets in the 
possession of r.e. subsets to the reducibilities which may hold between the two sets.

THEOREM 2. There exists a simple set $S$ and a pseudocreative set $P$ such that $P \leqq_{m} S$ but $S$ is not Turing reducible to $P$.

Proof. Let $S_{0}$ and $S_{1}$ be simple sets such that $S_{1}$ is not Turing reducible to $S_{0}$. (As mentioned in the proof of Corollary 2, such sets exist.) Let $S=J\left(S_{0}, S_{1}\right)$ and let $P$ be a cylinder of $S_{0}$. By Lemma 1 $S$ is simple, and as we mentioned above, $P$ is pseudocreative. Since $S_{0} \equiv_{m} P$ and $S_{0} \leqq_{1} S, P \leqq_{m} S$. On the other hand, $S_{1} \leqq_{1} S$, so if $S$ were Turing reducible to $P, S_{1}$ would also be Turing reducible to $P$, and hence $S_{1}$ would be Turing reducible to $S_{0}$, a contradiction.

REMARK. Theorem 2 also holds with "hypersimple" replacing "simple." This follows from the fact, which we shall not prove here, that if $A$ and $B$ are hypersimple sets, $J(A, B)$ is hypersimple also. Furthermore, the join of two maximal sets is easily shown to be hyperhypersimple. (See [4] for a definition of maximal sets, [7] for a definition of hypersimple and hyperhypersimple sets.) Thus if not all maximal sets are Turing equivalent, then Theorem 2 holds with "hyperhypersimple" replacing "simple."

\section{REFERENCES}

1. J. C. E. Dekker, $A$ theorem on hypersimple sets, Proc. Amer. Math. Soc. 4 (1953), 495-501.

2. J. C. E. Dekker and J. Myhill, Recursive equivalence types, Univ. California Press, Berkeley, Calif., 1960, pp. 67-211.

3. R. Friedberg, Two recursively enumerable sets of incomparable degrees of unsolvability, Proc. Nat. Acad. Sci. U.S.A. 43 (1958), 236-238.

4. - Three theorems on recursive enumeration, J. Symbolic Logic 23 (1958), 309-316.

5. S. C. Kleene and E. L. Post, The upper semi-lattice of degrees of recursive unsolvability, Ann. of Math. (2) 59 (1954), 379-407.

6. J. Myhill, Recursive digraphs, splinters and cylinders, Math. Ann. 138 (1959), 211-218.

7. E. L. Post, Recursively enumerable sets of positive integers and their decision problems, Bull. Amer. Math. Soc. 50 (1944), 284-316.

8. H. Rogers, Jr., Recursive functions and effective computability, McGraw-Hill, New York (to appear).

9. P. R. Young, On the structure of recursively enumerable sets, Doctoral dissertation, Massachusetts Institute of Technology, Cambridge, Mass., 1963.

Reed College

2 In a forthcoming paper, $A$ maximal set which is not complete, G. Sacks shows that (1) there are infinitely many r.e. Turing degrees which are degrees of maximal sets, and (2) a set is recursive if and only if it is recursive in every maximal set. 